\title{
Continuum modelling of gigahertz nano-oscillators
}

\author{
Barry J. Cox, Tamsyn A. Hilder, \\ Duangkamon Baowan, \\ Ngamta Thamwattana* \\ and James M. Hill
}

\author{
Nanomechanics Group, \\ School of Mathematics and Applied Statistics, \\ University of Wollongong, \\ Northfields Avenue, Wollongong, NSW 2522, Australia \\ Fax:+61242214845_E-mail: barryc@uow.edu.au \\ E-mail: tah429@uow.edu.au_E-mail: db898@uow.edu.au \\ E-mail:ngamta@uow.edu.au_E-mail: jhill@uow.edu.au
}

${ }^{*}$ Corresponding author

\begin{abstract}
Fullerenes and carbon nanotubes are of considerable interest throughout many scientific areas due to their unique and exceptional properties, such as low weight, high strength, flexibility, high thermal conductivity and chemical stability. These nanostructures have many potential applications in nano-devices. One concept that has attracted much attention is the creation of nano-oscillators, which can produce frequencies in the gigahertz range, for applications such as ultra-fast optical filters and nano-antennae. In this paper, we provide the underlying mechanisms of the gigahertz nano-oscillators and we review some recent results derived by the authors using the Lennard-Jones potential together with the continuum approach to mathematically model three different types of nano-oscillators including double-walled carbon nanotube, $\mathrm{C}_{60}$-nanotube and $\mathrm{C}_{60}$-nanotorus oscillators.
\end{abstract}

Keywords: nano-oscillator; double-walled carbon nanotube; $\mathrm{C}_{60}$ fullerene; nanotorus; Lennard-Jones potential.

Reference to this paper should be made as follows: Cox, B.J., Hilder, T.A., Baowan, D., Thamwattana, N. and Hill, J.M. (2008) 'Continuum modelling of gigahertz nano-oscillators', Int. J. Nanotechnol., Vol. 5, Nos. 2/3, pp.195-217.

Biographical notes: Barry J. Cox completed a Bachelor of Mathematics at the University of Wollongong graduating in 1989. In 2005, he enrolled in a Master of Mathematics by Research in the area of Mathematical Modelling in Nanotechnology, and in 2006 converted this enrolment to a $\mathrm{PhD}$. His interests include the mathematical modelling of electrorheological fluids and the mechanics and geometric structures of carbon nanotubes and fullerenes.

Tamsyn A. Hilder completed a Bachelor of Engineering Science with First Class Honours at Auckland University in 2001. She then worked as a Finite Element Analyst in both Australia and New Zealand for three years, with the main areas of focus being the automotive, rail and marine industries. In July 2005 she began her $\mathrm{PhD}$ in the area of Mathematical Modelling of Carbon Nanotubes and related nanostructures. 
Duangkamon Baowan finished her Bachelor Degree in Mathematics with First Class Honours in 2005 from Mahidol University, Thailand. She started her $\mathrm{PhD}$ in July 2005 on the topic of Modelling Multi-Walled Carbon Nanotubes as transversely isotropic elastic solids and the dynamics of gigahertz oscillators.

Ngamta Thamwattana received a Bachelor of Science with First Class Honours in Mathematics at Mahidol University, Thailand, in 2000. In 2001, she undertook her $\mathrm{PhD}$ in Applied Mathematics at the University of Wollongong, Australia and graduated in 2005. Her PhD focused on the Mechanics of Granular Materials. She is now an Australian Research Council Australian Postdoctoral Fellow in the Nanomechanics Group, University of Wollongong, working on nanotechnology. Her particular interests include mathematical modelling for gigahertz nano-oscillators and electrorheological nanofluids.

James M. Hill is a Professor of Theoretical Mechanics at the University of Wollongong. He has received two five year fellowships from the Australian Research Council; an ARC Senior Research Fellowship in 1997 to work on Granular Materials, and an ARC Australian Professorial Fellowship in 2004 to work on Nanomechanics. Since 1983 he has received 13 major research awards, including ARC Large Grants, ARC Discovery Projects, National Research Fellowship, National Teaching Company Scheme. He has published five books, and over 200 research publications in Applied Mathematics and Theoretical Mechanics. He is a Fellow of the Institute of Mathematics and its Applications. He has been an Associate Editor since 1982 of the ANZIAM Journal of Industrial and Applied Mathematics, which is published by the Australian Mathematical Society. His work has received international recognition through his appointment to the Editorial Boards of four international journals: Institute of Mathematics and its Applications, Journal of Applied Mathematics and the Quarterly Journal of Mechanics and Applied Mathematics, both published by Oxford University Press, Journal of Engineering Mathematics published by Kluwer Academic Press and Mathematics and Mechanics of Solids published by Sage Science Press.

\section{Introduction}

Both single- and multi-walled carbon nanotubes have been extensively studied by researchers from many disciplines aiming to create new nano-devices or to employ these structures for applications, such as labs on chips and targeted drug and gene delivery. This is due to their unique mechanical and electrical properties, and we refer the reader to Dresselhaus et al. [1], Harris [2], Rao et al. [3] and Qian et al. [4] for details of these properties for both single- and multi-walled carbon nanotubes. Recently, it has been found that the oscillation of the inner tube of a multi-walled carbon nanotube can generate frequencies in the gigahertz range, providing the potential for devices such as ultra-fast optical filters and ultra-sensitive nano-antennae. In this paper, we review these developments and summarise the authors' recent work on the mathematical models for three types of gigahertz oscillators, which are a double-walled carbon nanotube oscillator, a $\mathrm{C}_{60}$-nanotube oscillator and a $\mathrm{C}_{60}$-nanotorus oscillator/orbiter. We note that the last configuration is novel and has not been studied previously, either experimentally or theoretically. While research in this area is dominated by experimental and highly computational molecular dynamics studies, we emphasise here the use of elementary 
mechanics and applied mathematical modelling techniques to generate results that are comparable to those of molecular dynamics simulations.

The discovery of double-walled carbon nanotube oscillators originated with experiments by Cumings and Zettl [5] on multi-walled carbon nanotubes, where they remove the cap from one end of the outer shell and attach a moveable nanomanipulator to the core in a high-resolution transmission electron microscope. By pulling the core out and pushing it back into the outer shell, they report an ultra-low sliding frictional force. This result is also confirmed by Yu et al. [6]. Further, Cumings and Zettl [5] also observe that the extruded core, after release, quickly and fully retracts inside the outer shell due to the restoring force resulting from the van der Waals interaction acting on the extruded core. These results led Zheng and Jiang [7] and Zheng et al. [8] to study the molecular gigahertz oscillators, where the sliding of the inner shell inside the outer shell of a multi-walled carbon nanotube can generate oscillatory frequencies up to several gigahertz. They consider this problem by using the Lennard-Jones potential and Newton's second law to calculate the frequency of oscillation. They find that the oscillatory frequency is in the gigahertz range and their results also support the experimental findings of Cumings and Zettl [5] that the frictional effect of the intershell sliding is negligibly small. Moreover, this phenomenon is confirmed by molecular dynamics simulations of Legoas et al. [9] and Rivera et al. [10,11]. In Baowan and Hill [12], the force distribution for double-walled carbon nanotubes and the oscillation of the inner tube are investigated. This study uses the continuum approach of the Lennard-Jones potential and Newton's second law, where the frictional force is neglected. They obtain an exact analytical expression for the interaction energy between the inner and outer tubes and the van der Waals interaction force. This model also predicts the gigahertz frequency oscillatory behaviour of the double-walled carbon nanotube oscillator.

Regarding the frequency of oscillation, Zheng et al. [8] observe that the shorter the inner tube, the higher the frequency. Instead of using an inner tube, Liu et al. [13] find that a higher frequency can be generated by the oscillation of a $\mathrm{C}_{60}$ fullerene inside a single-walled carbon nanotube. For further details of fullerenes, we refer the reader to Dresselhaus et al. [1]. While Liu et al. [13] and Qian et al. [14] study a $\mathrm{C}_{60}$-nanotube oscillator using molecular dynamics simulations, Cox et al. $[15,16]$ employ elementary mechanical principles, utilising the continuum approach to provide a classical applied mathematical model. In particular, Cox et al. [15] express analytically the suction energy of a $\mathrm{C}_{60}$ molecule upon entering a carbon nanotube. This energy is imparted onto the $\mathrm{C}_{60}$ molecule in terms of kinetic energy and thus induces the oscillating motion. These authors also determine the condition on the radius of carbon nanotubes that will accept the $\mathrm{C}_{60}$ molecule at rest in the vicinity of an open end of the tube. Further, a novel mathematical model for the oscillation of the $\mathrm{C}_{60}$ fullerene inside a single-walled carbon nanotube is shown in Cox et al. [16], where the axial van der Waals restoring force of the $\mathrm{C}_{60}$ molecule is approximated by two equal and opposite Dirac delta functions operating at both ends of the nanotube. The results of the classical mathematical model of Cox et al. $[15,16]$ are shown to be in good agreement with numerical results of Girifalco et al. [17] and Hodak and Girifalco [18], and molecular dynamics simulations of Liu et al. [13] and Qian et al. [14].

There may exist many such oscillators at the nanoscale, and Hilder and Hill [19] examine the possibility of creating a $\mathrm{C}_{60}$-nanotorus oscillator/orbiter, where a $\mathrm{C}_{60}$ fullerene orbits inside a carbon nanotorus. Liu et al. [20] observe single continuous toroidal carbon nanotubes, and subsequently term them fullerene 'crop circles'. 
Martel et al. [21] succeed in forming rings using straight single-walled carbon nanotubes, where the ring circumference is equal to the initial tube length. Effectively, the toroidal nanotube structure can be viewed as a single-walled carbon nanotube closed around onto itself to form a perfect torus. In this paper these toroidal structures are referred to as nanotori.

Creation of a $\mathrm{C}_{60}$-nanotorus oscillator/orbiter may involve either closing a nanotube already containing an oscillating buckyball into a torus; injecting a buckyball into the torus just prior to closure; or closing a nanotube containing a stationary buckyball to form a torus and subsequently initialising the oscillation by some applied external field (electric, magnetic or chemical doping), thereby inducing a velocity on the enclosed buckyball. These procedures pose numerous practical challenges that need to be overcome before an actual $\mathrm{C}_{60}$-nanotorus oscillator can be realised. The ultra-low friction effect demonstrated by Cumings and Zettl [5] may also be exhibited in the $\mathrm{C}_{60}$-nanotorus oscillator, and if so, the buckyball might orbit almost indefinitely inside the nanotorus. A sealed structure is ideal in terms of working devices, and the $\mathrm{C}_{60}$-nanotorus oscillator may well be the ultimate oscillator. As far as the authors are aware a $\mathrm{C}_{60}$-nanotorus oscillator has yet to be constructed. Hilder and Hill [19] aim to assess its feasibility by considering the basic mechanics of such a system. Regardless of the speculative nature of these potential nanoscale devices, such a study must inevitably precede any practical implementation.

In this paper, we review the work of Baowan and Hill [12], Cox et al. [15,16] and Hilder and Hill [19], and summarise the essential mechanisms of the three types of gigahertz nano-oscillators. In the following section, we introduce the Lennard-Jones potential for the non-bonded interaction energy between two molecules. Using the continuum approach, we determine the interaction energies of the inner and outer shells of a double-walled carbon nanotube, $\mathrm{a}_{60}$ fullerene located inside a single-walled carbon nanotube and a $\mathrm{C}_{60}$ fullerene inside a carbon nanotorus. From the potential energies of each configuration, the van der Waals restoring forces are obtained, which are then used in Sections 3-5 to describe the oscillatory behaviours of the three nano-oscillators, respectively.

\section{Interaction energy}

The non-bonded interaction energy between two carbon molecules can be obtained by summing the potential interaction for each atom pair

$$
E=\sum_{i} \sum_{j} \Phi\left(r_{i j}\right),
$$

where $\Phi\left(r_{i j}\right)$ is a potential function for atoms $i$ and $j$ on each molecule a distance $r_{i j}$ apart. Following Girifalco et al. [17] and Hodak and Girifalco [18], we adopt a continuum approach where carbon atoms are assumed to be uniformly distributed over the surfaces of the molecules. Thus, instead of the double summation in equation (1), the interaction energy can be obtained equivalently by performing double surface integrals, averaging over the surface of each entity

$$
E=n_{1} n_{2} \iint \Phi(r) \mathrm{d} \Sigma_{1} \mathrm{~d} \Sigma_{2},
$$


where $n_{1}$ and $n_{2}$ are the mean surface density of atoms on each molecule and $r$ denotes the distance between two typical surface elements $d \Sigma_{1}$ and $d \Sigma_{2}$ on each molecule. In this paper, we adopt the Lennard-Jones potential given by

$$
\Phi(r)=-A r^{-6}+B r^{-12},
$$

where $A$ and $B$ are the attractive and repulsive constants, respectively. We note that there are a number of empirically motivated models of potential in the literature, for example, the Morse potential is used in Wang et al. [22] for the problem of two $\mathrm{C}_{60}$ fullerenes in contact. We refer the reader to Qian et al. [4] and Rieth [23] for details of the other models and their applications. Alternatively, the Lennard-Jones potential (equation (3)) can be written in the form

$$
\Phi(r)=4 \omega\left[-\left(\frac{\sigma}{r}\right)^{6}+\left(\frac{\sigma}{r}\right)^{12}\right],
$$

where $\sigma$ is the van der Waals diameter and $\omega$ denotes the energy well depth, $\omega=A^{2} /(4 B)$. From equation (4), the equilibrium distance $r_{0}$ for two atoms is given by $r_{0}=2^{1 / 6} \sigma=(2 B / A)^{1 / 6}$.

The Lennard-Jones potential has been used in a number of studies to determine the van der Waals interaction energy and force for two carbon nanostructures. Girifalco [24] determines the interaction energy between two $\mathrm{C}_{60}$ fullerenes, and then extend the study in Girifalco et al. [17] to find the energy between two identical parallel carbon nanotubes of infinite length and between a carbon nanotube and a $\mathrm{C}_{60}$ fullerene (both inside and outside the tube). Girifalco et al. [17] also provide the value of the interaction constants in the Lennard-Jones potential for carbon atoms in graphene-graphene, $\mathrm{C}_{60}-\mathrm{C}_{60}$ and $\mathrm{C}_{60}$-graphene, as shown in Table 1. Further, Hodak and Girifalco [18] propose an energy formula for universal graphitic systems, which also include the interaction of an ellipsoid inside a single-walled carbon nanotube. Henrard et al. [25] use a similar technique to Girifalco [24] and obtain the potential for single-walled carbon nanotubes in bundles. Following Henrard et al. [25] and Girifalco et al. [17], Zheng et al. [8] use the Lennard-Jones potential to determine the van der Waals restoring force between the inner and outer shells of a multi-walled carbon nanotube and subsequently predict a gigahertz frequency of the oscillatory motion.

Table 1 Lennard-Jones constants in the graphitic systems (Girifalco et al. [17])

\begin{tabular}{lcccc}
\hline & $A\left(\mathrm{eV} \times \AA^{6}\right)$ & $B\left(\mathrm{eV} \times \AA^{12}\right)$ & $r_{0}(\AA)$ & $|\omega|(\mathrm{meV})$ \\
\hline Graphene-graphene & 15.2 & $24.1 \times 10^{3}$ & 3.83 & 2.39 \\
$\mathrm{C}_{60}-\mathrm{C}_{60}$ & 20.0 & $34.8 \times 10^{3}$ & 3.89 & 2.86 \\
$\mathrm{C}_{60}$-graphene & 17.4 & $29.0 \times 10^{3}$ & 3.86 & 2.62 \\
\hline
\end{tabular}

We note that the integral (2) can be difficult to evaluate, particularly for certain configurations, such as an ellipsoidal fullerene inside (or outside) a carbon nanotube and a $\mathrm{C}_{60}$ fullerene interacting with a carbon nanotorus. While Girifalco et al. [17], Hodak and Girifalco [18], Zheng et al. [8] and others express the potential energy in terms of integral forms and subsequently perform their analysis using numerical integration, the authors $[12,15,16,19]$ perform these integrations analytically and express the potential in closed 
form in terms of hypergeometric and other special functions. Furthermore, the authors' choice of using the continuum approach rather than the discrete atom-atom model is justified as pointed out by Girifalco et al. [17] that from a physical point of view both discrete (e.g., molecular dynamics simulations) and continuum models (e.g., $[12,15-19,24])$ make assumptions that are incorrect, but it can be argued that the continuum model is closer to reality.

In the following subsections, we look at the interaction between the following carbon nanostructures; inner-outer tubes of a double-walled carbon nanotube, $\mathrm{C}_{60}$-nanotube and $\mathrm{C}_{60}$-nanotorus. These results are then used in subsequent sections to determine the van der Waals restoring forces and the oscillatory frequencies.

\subsection{Interaction between the inner and outer tubes of a double-walled carbon nanotube}

In this subsection we determine the potential energy $E$ between the inner and outer tubes of a double-walled carbon nanotube using the continuum approximation as described in Section 2. With reference to the rectangular Cartesian coordinate system, the inner and outer tubes are in the same co-axial direction with parametric equations $\left(a \cos \theta_{1}, a \sin \theta_{1}, z_{1}\right)$ and $\left(b \cos \theta_{2}, b \sin \theta_{2}, z_{2}\right)$ respectively. As shown in Figure $1, a$ and $b$ denote radii of the inner and outer tubes, respectively, $-\pi \leq \theta_{1}, \theta_{2} \leq \pi,-L_{1} \leq z_{1} \leq L_{1}$ and $-L_{2} \leq z_{2} \leq L_{2}$. From Figure 1, the distance $\rho$ is given by

$$
\rho^{2}=\left(b \cos \theta_{2}-a \cos \theta_{1}\right)^{2}+\left(b \sin \theta_{2}-a \sin \theta_{1}\right)^{2}+\left(z_{2}-z_{1}\right)^{2} .
$$

Figure 1 Double-walled carbon nanotube with the inner and outer tubes of lengths $2 L_{1}$ and $2 L_{2}$, respectively

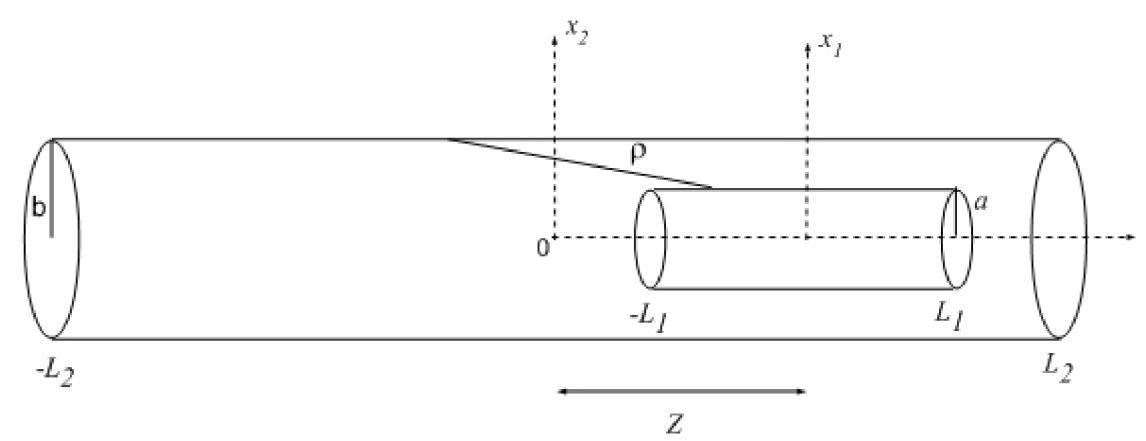

Thus, by performing the integrals shown in equation (2) we obtain the total potential energy $E$ for the inner and outer shells of a double-walled carbon nanotube, namely

$$
E(Z)=8 \pi n_{g}^{2} a b\left(-A N_{6}+B N_{12}\right)
$$

where $n_{g}$ is the mean surface density of carbon atoms on a nanotube, and $N_{6}$ and $N_{12}$ are given by 


$$
\begin{aligned}
& N_{6}=\sum_{i=1}^{4}(-1)^{i+1} {\left[\frac{3 \pi}{16} \sum_{k=0}^{\infty} \frac{(2 k) !\left(Z-\beta_{i}\right)^{2 k+2}}{2^{2 k}(k !)^{2}(2 k+1)(a+b)^{5}\left[(a+b)^{2}+\left(Z-\beta_{i}\right)^{2}\right]^{k+1 / 2}}\right.} \\
& \quad \times F_{1}\left(\frac{1}{2} ; \frac{5}{2}, k+\frac{1}{2} ; 1 ; \frac{4 a b}{(a+b)^{2}}, \frac{4 a b}{(a+b)^{2}+\left(Z-\beta_{i}\right)^{2}}\right) \\
&-\left.\frac{\pi}{16(a+b)^{2}\left[(a+b)^{2}+\left(Z-\beta_{i}\right)^{2}\right]} F_{1}\left(\frac{1}{2} ; 1,1 ; 1, \frac{4 a b}{(a+b)^{2}}, \frac{4 a b}{(a+b)^{2}+\left(Z-\beta_{i}\right)^{2}}\right)\right], \\
& N_{12}=\sum_{i=1}^{4}(-1)^{i+1}\left[\frac{63 \pi}{512} \sum_{k=0}^{\infty} \frac{2^{2 k}(k !)^{2}(2 k+1)(a+b)^{11}\left[(a+b)^{2}+\left(Z-\beta_{i}\right)^{2}\right]^{k+1 / 2}}{2}\right) \times F_{1}\left(\frac{1}{2} ; \frac{11}{2}, k+\frac{1}{2} ; 1 ; \frac{4 a b}{(a+b)^{2}}, \frac{4 a b}{(a+b)^{2}+\left(Z-\beta_{i}\right)^{2}}\right) \\
&- \frac{21 \pi}{512(a+b)^{8}\left[(a+b)^{2}+\left(Z-\beta_{i}\right)^{2}\right]} F_{1}\left(\frac{1}{2} ; 4,1 ; 1, \frac{4 a b}{(a+b)^{2}}, \frac{4 a b}{(a+b)^{2}+\left(Z-\beta_{i}\right)^{2}}\right) \\
&- \frac{21 \pi}{1280(a+b)^{6}\left[(a+b)^{2}+\left(Z-\beta_{i}\right)^{2}\right]^{2}} F_{1}\left(\frac{1}{2} ; 3,2 ; 1, \frac{4 a b}{(a+b)^{2}}, \frac{4 a b}{(a+b)^{2}+\left(Z-\beta_{i}\right)^{2}}\right) \\
&- \frac{3 \pi}{320(a+b)^{4}\left[(a+b)^{2}+\left(Z-\beta_{i}\right)^{2}\right]^{3}} F_{1}\left(\frac{1}{2} ; 2,3 ; 1, \frac{4 a b}{(a+b)^{2}}, \frac{4 a b}{(a+b)^{2}+\left(Z-\beta_{i}\right)^{2}}\right) \\
&\left.-\frac{\pi}{160(a+b)^{2}\left[(a+b)^{2}+\left(Z-\beta_{i}\right)^{2}\right]^{4}} F_{1}\left(\frac{1}{2} ; 1,4 ; 1, \frac{4 a b}{(a+b)^{2}}, \frac{4 a b}{(a+b)^{2}+\left(Z-\beta_{i}\right)^{2}}\right)\right]
\end{aligned}
$$

where $F_{1}\left(\alpha ; \beta, \beta_{1} ; \gamma ; x, y\right)$ is an Appell's hypergeometric function of two variables [26-28] and $\beta_{1}=-L_{1}-L_{2}, \beta_{2}=L_{1}-L_{2}, \beta_{3}=L_{1}+L_{2}$, and $\beta_{4}=-L_{1}+L_{2}$. These lengthy expressions (7) and (8) may be readily evaluated using the algebraic computer package MAPLE. We note that positions $\beta_{1}, \beta_{2}, \beta_{3}$ and $\beta_{4}$ as depicted in Figure 2 are the four critical positions for the two concentric carbon nanotubes, which give rise to an interesting behaviour for the potential energy and force distribution, as discussed in Baowan and Hill [12] and also in Section 3. For full details of the mathematical derivations, we also refer the reader to Baowan and Hill [12]. In Section 3, the potential $E$ as shown in equation (6) is used to determine the van der Waals restoring force for the oscillatory behaviour of double-walled carbon nanotube oscillators.

\subsection{Interaction of $C_{60}$ with a single-walled carbon nanotube}

In axially symmetric cylindrical polar coordinates, we assume that the $\mathrm{C}_{60}$ fullerene of radius $b$ is located at $(\varepsilon, 0,0)$, as shown in Figure 3 , in a carbon nanotube of infinite extent with a parametric equation $(a \cos \theta, a \sin \theta, z)$. We note that $\varepsilon$ is the distance between the centre of the offset $\mathrm{C}_{60}$ fullerene and the central axis of the tube, $a$ is the tube radius, $-\pi \leq \theta \leq \pi$ and $-\infty<z<\infty$. 
Figure 2 Four critical positions for two concentric carbon nanotubes

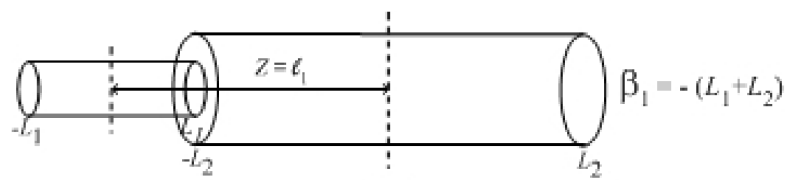

(a)

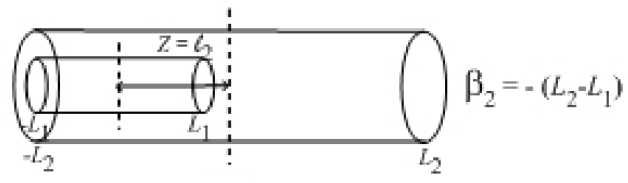

(b)

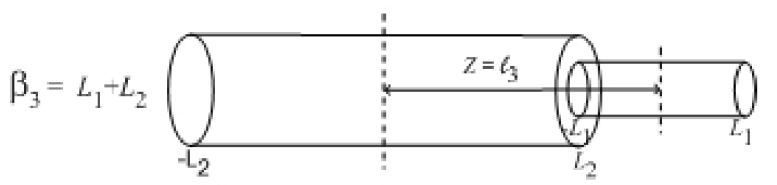

(c)

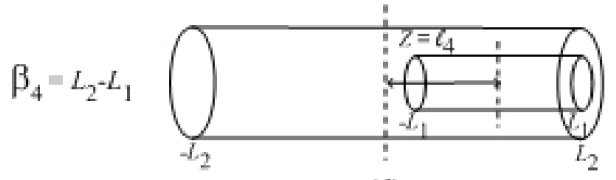

(d)

Figure 3 Offset $\mathrm{C}_{60}$ fullerene inside a single-walled carbon nanotube

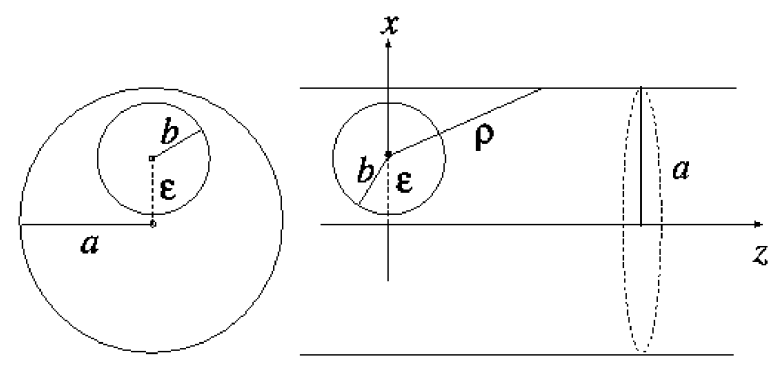

In this subsection, we determine the potential energy of a $\mathrm{C}_{60}$ fullerene inside a single-walled carbon nanotube and its preferred position with reference to the cross-section of the tube. The preferred position is where the molecule admits the minimum potential energy. From Figure 3, we find $\rho=\left(a^{2}+\varepsilon^{2}-2 a \varepsilon \cos \theta+z^{2}\right)^{1 / 2}$, thus by performing the integrals shown in equation (2) over the entire surfaces of the $\mathrm{C}_{60}$ fullerene and the carbon nanotube, we obtain the potential energy $E$ for the offset $\mathrm{C}_{60}$ fullerene inside the carbon nanotube, namely

$$
\begin{aligned}
E= & 4 \pi^{2} a b^{2} n_{f} n_{g}\left[-\frac{A}{8}\left(3 I_{2}+5 b^{2} I_{3}\right)+\frac{B}{5}\left(\frac{315}{256} I_{5}\right.\right. \\
& \left.\left.+\frac{1155 b^{2}}{64} I_{6}+\frac{9009 b^{4}}{128} I_{7}+\frac{6435 b^{6}}{64} I_{8}+\frac{12155 b^{8}}{256} I_{9}\right)\right],
\end{aligned}
$$


where $n_{f}$ and $n_{g}$ denote the mean surface density of carbon atoms on a buckyball and a carbon nanotube respectively, and the integrals $I_{n}$ are defined by

$$
I_{n}=\int_{-\pi}^{\pi}(\alpha-\beta \cos \theta)^{-(n+1 / 2)} \mathrm{d} \theta
$$

and $\alpha=a^{2}+\varepsilon^{2}-b^{2}$ and $\beta=2 a \varepsilon$. We note that the integrals $I_{n}$ can be evaluated in terms of elliptic integrals or in terms of hypergeometric functions (see Cox et al. [16]). Also, we refer to $[15,16]$ for the full mathematical derivations.

In Figure 4, the potential energy $E$ is plotted with respect to the radial distance $\varepsilon$ from the tube axis. It can be seen that the preferred position for the $\mathrm{C}_{60}$ fullerene inside a $(10,10)$ carbon nanotube $(a=6.784 \AA)$ is where the centre of the $\mathrm{C}_{60}$ fullerene lies on the tube axis $(\varepsilon=0)$. For a $(16,16)$ carbon nanotube $(a=10.856 \AA)$, we find $\varepsilon=4.314 \AA$. Further, we observe that as the tube radius gets larger, the location where the minimum energy occurs tends to be closer to the nanotube wall. These results agree with the findings of Girifalco et al. [17].

Figure 4 Potential of an offset $\mathrm{C}_{60}$ fullerene inside a $(10,10)$ and $(16,16)$ carbon nanotube, with respect to distance $\varepsilon$

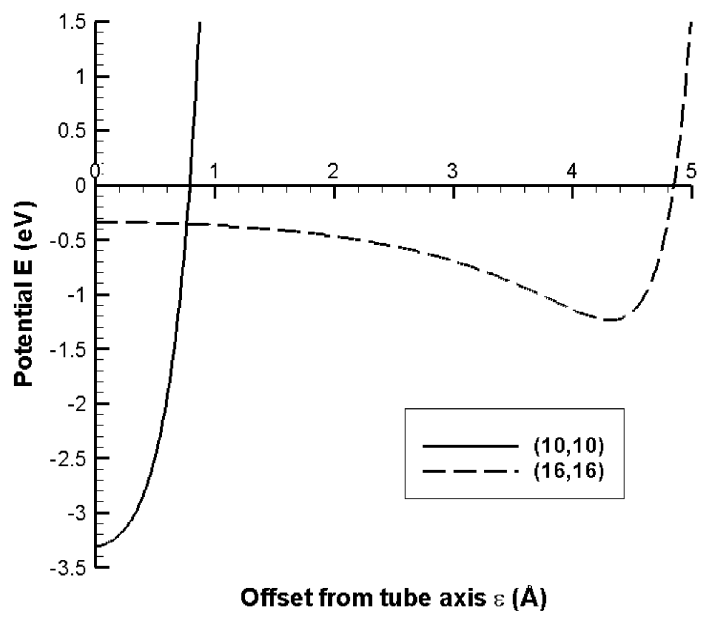

\subsection{Interaction of $C_{60}$ inside a single-walled carbon nanotorus}

Here we determine the potential energy for a $\mathrm{C}_{60}$ fullerene orbiting inside a carbon nanotorus. The position of the $\mathrm{C}_{60}$ fullerene centre is illustrated in Figure 5 and defined by

$$
x=\left(c+\varepsilon \cos \varphi_{1}\right) \cos \theta_{1}, \quad y=\left(c+\varepsilon \cos \varphi_{1}\right) \sin \theta_{1}, \quad z=\varepsilon \sin \varphi_{1},
$$

where $c$ is the nanotorus ring radius taken as $1500 \AA$ [20], $b$ is the nanotorus tube radius and the centre of the buckyball is a distance $\varepsilon$ from the cross-sectional tube centre, at an angle $\varphi_{1}$ from the horizontal. The radius of the buckyball $a$, is taken to be $3.55 \AA$ [18]. 
Figure $5 \quad \mathrm{C}_{60}$-nanotorus oscillator and its coordinates
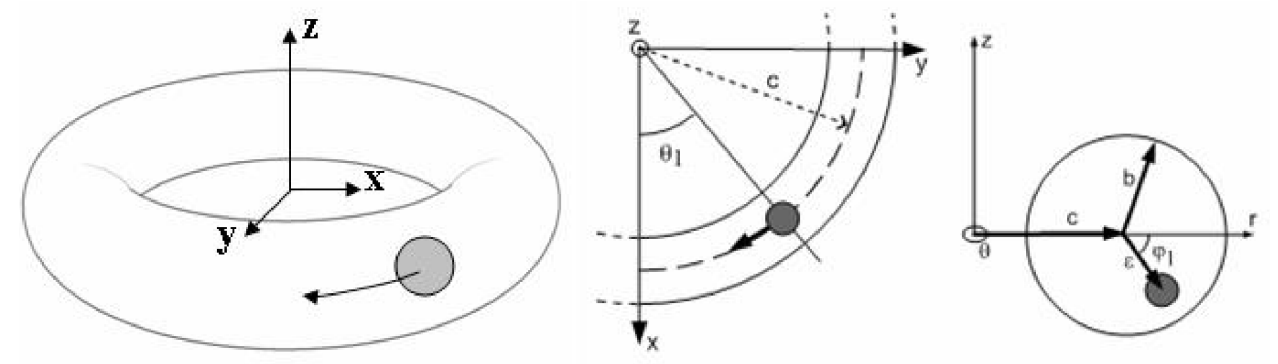

Using equations (2) and (3) we can evaluate the Lennard-Jones energy between the buckyball and the nanotorus,

$$
\begin{aligned}
E= & 256 \pi^{3} a b c n_{f} n_{g}\left[\frac{-5 A a^{3} c^{3}(c+\varepsilon)^{3}}{2[\delta(4 c(c+\varepsilon)+\delta)]^{7 / 2}} F_{1}\left(\frac{1}{2} ; \frac{7}{2}, \frac{7}{2} ; 1 ; \alpha, \beta\right)\right. \\
& \left.+\frac{155584 B a^{9} c^{9}(c+\varepsilon)^{9}}{[\delta(4 c(c+\varepsilon)+\delta)]^{1 / 2}} F_{1}\left(\frac{1}{2} ; \frac{19}{2}, \frac{19}{2} ; 1 ; \alpha, \beta\right)\right],
\end{aligned}
$$

where $n_{f}$ and $n_{g}$ are respectively the mean surface densities of carbon atoms on the buckyball and nanotorus (assumed to be created from closing a carbon nanotube), $\delta=(b+\varepsilon)^{2}-a^{2}, \alpha=4 b \varepsilon / \delta$ and $\beta=4 b \varepsilon /[4 c(c+\varepsilon)+\delta]$. Note that the energy potential given above is only valid for $|\varepsilon| \leq|b-a|$ and $F_{1}\left(\alpha ; \beta, \beta_{1} ; \gamma ; x, y\right)$ is an Appell's hypergeometric function of two variables [26-28]. We refer the reader to Hilder and Hill [19] for full details of the mathematical derivations.

Using the algebraic computer package MAPLE, we plot the Lennard-Jones energy against buckyball position, shown in Figure 6 for both a nanotorus created from closing a $(10,10)(b=6.784 \AA)$ and a $(16,16)(b=10.856 \AA)$ nanotube. The gravity potential is found to be negligible when compared to the centrifugal and Lennard-Jones potentials, hence we assume $\varphi_{1}=0$. The buckyball's minimum energy or equilibrium position is found to depend on the nanotorus tube radius $b$, where the buckyball moves closer to the nanotorus wall as the radius increases. For example, the equilibrium position for the $(10,10)$ nanotorus is $\varepsilon=0.9 \AA$, while for the $(16,16)$ nanotorus we obtain $\varepsilon=5.25 \AA$. These represent distances from the tube wall of $2.334 \AA$ and $2.056 \AA$, respectively. A similar observation is made in Section 2.2 (Cox et al. [16]). The inclusion of the centrifugal potential moves the minimum energy position further from the centre of rotation. The position also depends on the angular velocity, where it is more dominant for larger velocities. In the limit as $c$ tends to infinity in equation (12), we obtain overall agreement with Cox et al. [16]. However, to obtain an equation for the Lennard-Jones energy of the buckyball in the nanotorus, only the leading order terms are included and as a result there is a distinction between the two models. 
Figure 6 Lennard-Jones energy with respect to the buckyball position $\mathcal{E}$, for $(10,10)$ and $(16,16)$ carbon nanotorus

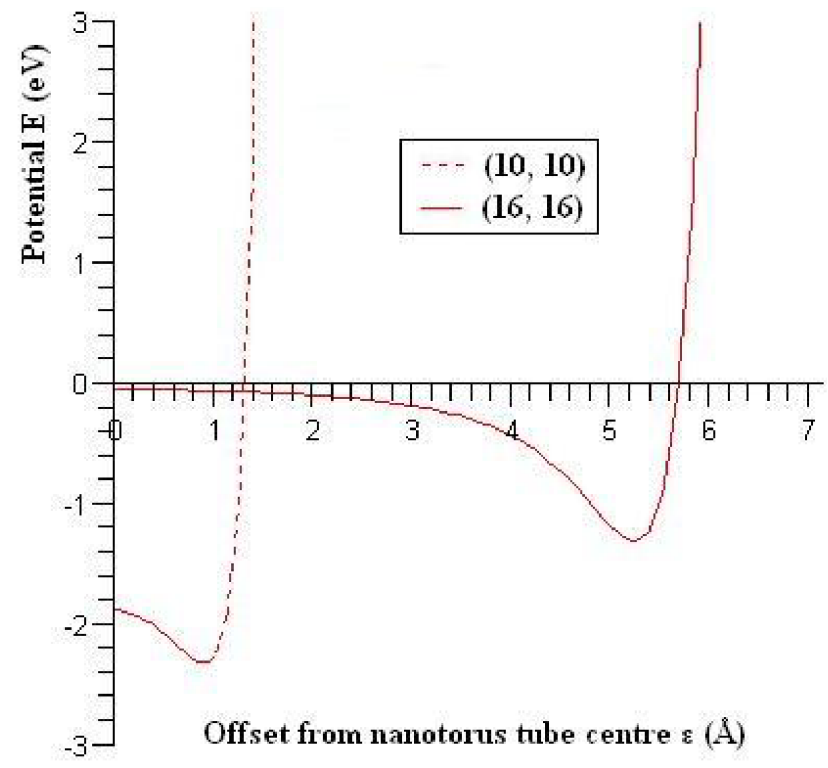

In the following sections, we use the Lennard-Jones potential energies to determine the van der Waals interaction forces and subsequently study the oscillatory behaviours of the three types of gigahertz oscillators.

\section{Double-walled carbon nanotube oscillators}

In order to study the oscillatory behaviour of double-walled carbon nanotubes, we use Newton's second law, which on neglecting the frictional force becomes

$$
M \frac{\mathrm{d}^{2} Z}{\mathrm{~d} t^{2}}=F_{Z}^{t o t}(Z)=-\frac{\partial E}{\partial Z}
$$

where $M$ is the mass of the inner tube. Following Cumings and Zettl [5], we pull the inner tube out a distance $d$ and release it, so that the distance between their centres becomes $Z_{0}=L_{2}-L_{1}+d$, which leads to the geometric constraint $d \leq 2 L_{1}$. Using the equations of motion, for the particular case that the initial velocity is zero, the oscillation frequency may be written

$$
f=\frac{\alpha \sqrt{d}}{4\left(2 d+\beta_{4}\right)},
$$

where $\alpha^{2}=|E(0)| /\left(M L_{1}\right)$ and $E(0)$ is the potential energy at $Z=0$. We note that the equation (14) has a maximum value for $d=\left(L_{2}-L_{1}\right) / 2$. We note that the case $L_{1}=L_{2}$ gives rise to small oscillations near a stable equilibrium point. The oscillation period is very short and as a result, we obtain the maximum frequency value. 
From Girifalco et al. [17], Hodak and Girifalco [18] and Ma et al. [29], we obtain the constant values used in the model for double-walled carbon nanotube oscillators. By using the algebraic computer package MAPLE, we plot the potential energy and van der Waals force vs. the difference between the centres of the tubes $Z$, as shown in Figures 7-9 for variations of the inner tube lengths. As shown in Figure 7 for the case of equal lengths of the inner and outer tubes, the minimum potential energy occurs at $Z=0$, which is the position where the tubes overlap. Moreover, the forces at this point balance each other and therefore represent an equilibrium position. By perturbing the inner tube in the axial direction away from its preferential position $Z=0$, it will oscillate and quickly return to the position $Z=0$. On the other hand, if the overall inner tube is outside the outer tube, the repulsive forces at the ends of the outer tube will not allow the inner tube to get inside unless an initial force is provided to overcome these repulsive forces, Figure 7(c).

Figure 7 (a) Potential; (b) van der Waals force and (c) repulsive force, for a double-walled carbon nanotube of inner tube length $L_{1}=500 \AA$ and outer tube length $L_{2}=500 \AA$, where $Z$ represents the distance between centres of the tubes

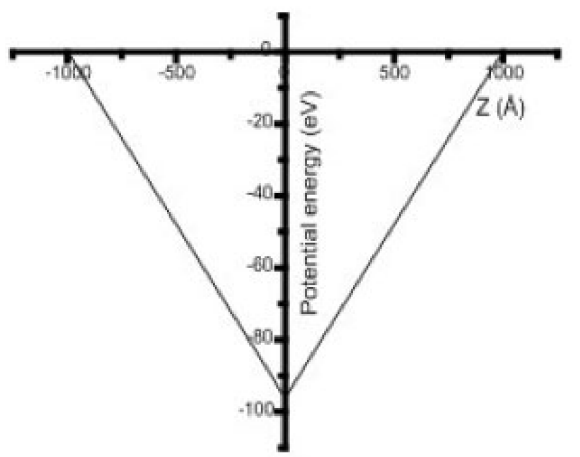

(a)

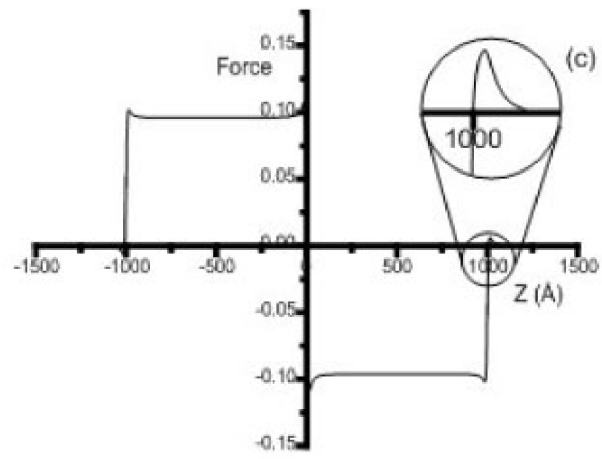

(b)

Figure 8 (a) Potential; (b) van der Waals force and (c) repulsive force, for a double-walled carbon nanotube of inner tube length $L_{1}=250 \AA$ and outer tube length $L_{2}=500 \AA$, where $Z$ represents the distance between centres of the tubes

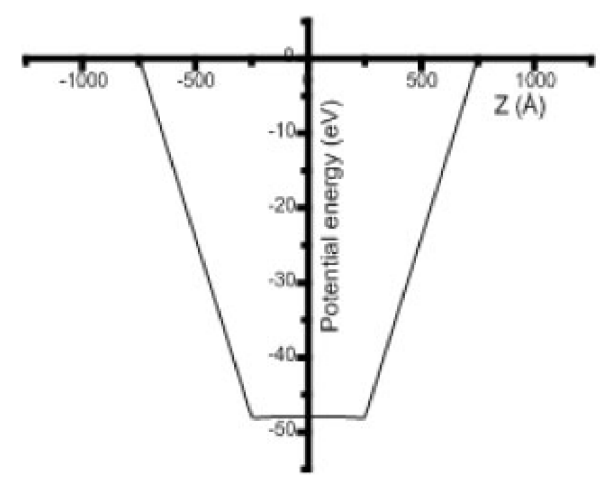

(a)

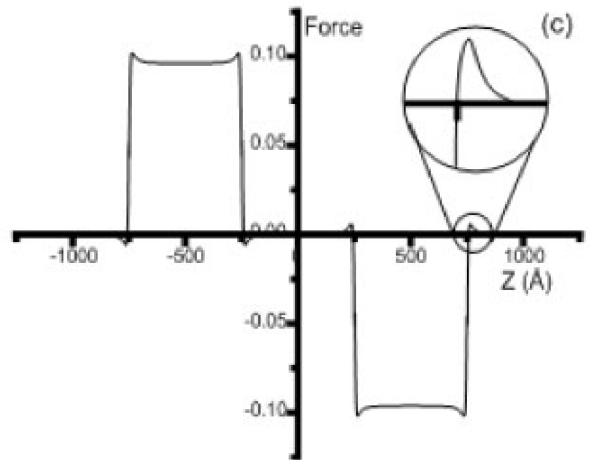

(b) 
Figure 9 (a) Potential and (b) van der Waals force, for a double-walled carbon nanotube of inner tube length $L_{1}=1 \AA$ and outer tube length $L_{2}=500 \AA$, where $Z$ represents the distance between centres of the tubes

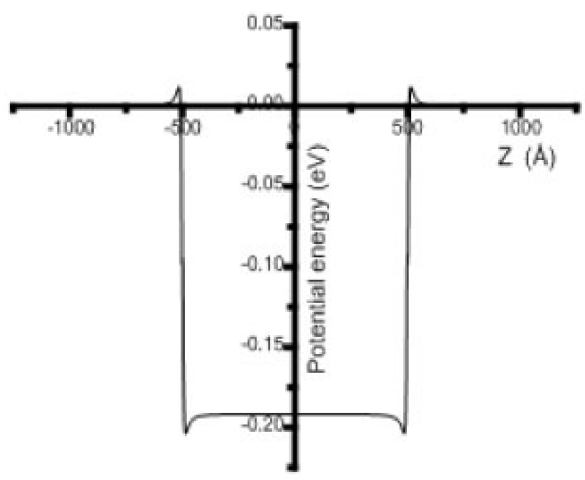

(a)

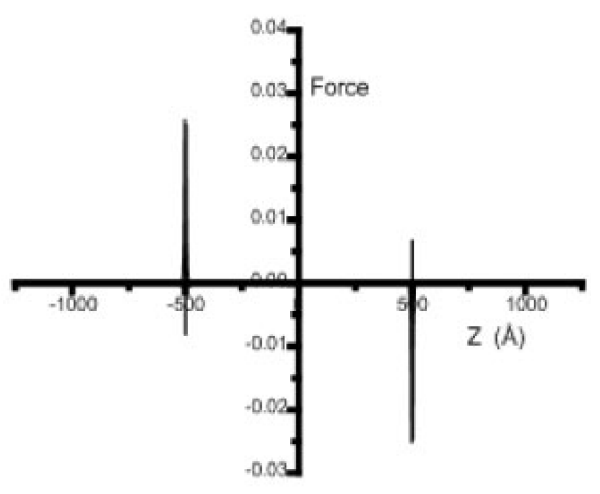

(b)

The potential energy and the force distribution for $L_{1}=250 \AA$ and $L_{2}=500 \AA$ are shown in Figure 8. The minimum potential energy is also at the position $Z=0$, and maintains this constant value in the range between $\beta_{2}$ to $\beta_{4}$ which are the two critical positions defined in Section 2.1. In terms of the van der Waals force, there are two attractive forces at both ends of the outer tube, which keep the inner tube oscillating. We note that the ends of each phase for the attractive forces are also the critical positions, $\beta_{1}$ and $\beta_{3}$. Again, the repulsive forces at the outer tube ends will not allow the inner tube inside. An initial force is then needed for an oscillation of the inner tube to occur.

Next, we further reduce the length of the inner tube. As $L_{1}$ becomes less than the inner tube radius $a$, the inner tube behaves like a buckyball. The minimum energy is everywhere along the outer tube and there are two strong attractive forces at the ends to keep the inner tube oscillating. As shown in Figure 9, we observe that the forces are close to zero everywhere except at both ends of the outer tube. Accordingly, these forces can be estimated by two equal and opposite Dirac delta functions, similar to the observation shown in Section 4 (Cox et al. [16]).

By using Newton's second law, we obtain an oscillation frequency for the double-walled carbon nanotube with the inner tube oscillating, as depicted in Figure 10. The inner tube is pulled out a distance $d$ from the end of the outer tube and released, the inner tube then moves back into the outer shell with the potential energy of the tubes and a kinetic energy resulting from an initial velocity. Moreover, there are repulsive and attractive forces at the ends of the outer tube to keep the inner tube in and maintain the oscillatory behaviour. In particular, for zero initial velocity the maximum frequency occurs when the inner tube is pulled out a distance $d=\left(L_{2}-L_{1}\right) / 2$. We comment that the frequency adopted in this context refers to the period $(T=2 \pi / f)$ for which the oscillating molecule travels at a constant velocity apart from the instantaneous reversal occurring at the tube extremities. 
Figure 10 Frequency profile for inner tube with varying lengths vs. the extrusion distance $d$ (for colours see online version)

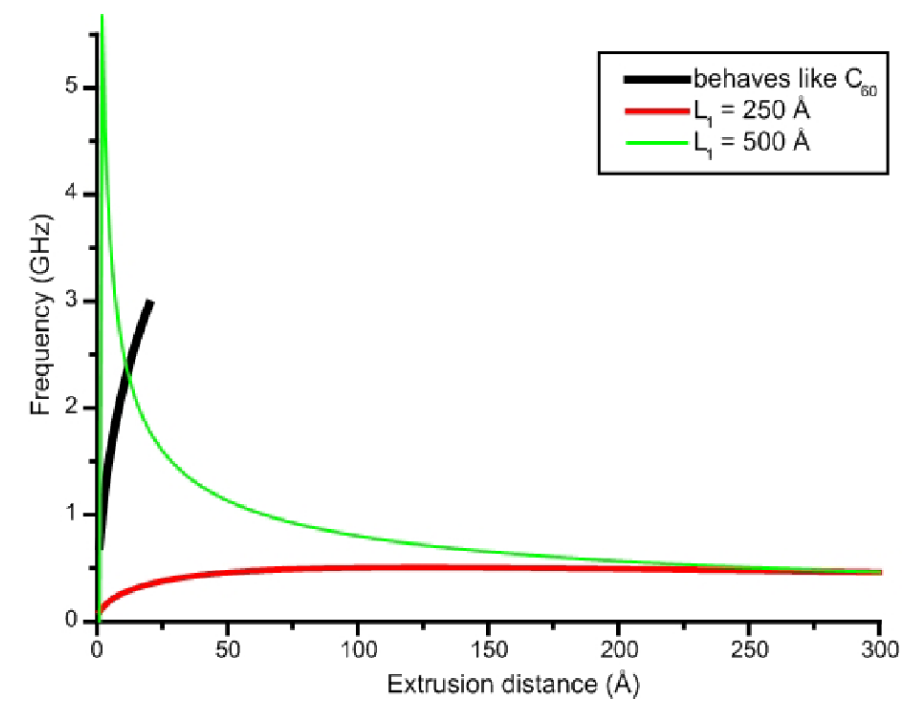

\section{$4 \quad \mathrm{C}_{60}$ single-walled carbon nanotube oscillators}

In this section, we review Cox et al. [15,16] and summarise the underlying mechanisms of an oscillating $\mathrm{C}_{60}$ molecule inside a single-walled carbon nanotube. From the molecular dynamics study of Qian et al. [14], it is shown that a $\mathrm{C}_{60}$ molecule located on the axis of a nanotube and in vicinity of the tube's open end will get sucked into the tube and begin the oscillatory motion spontaneously. As such, in this section we first deal with an issue of determining the suction and repulsion condition for a $\mathrm{C}_{60}$ molecule locating near an open end of a carbon nanotube. In the second part, we consider the fullerene which gets sucked in by the nanotube and thus oscillates between the tube ends.

\subsection{Acceptance and suction energies}

In this subsection, we investigate the suction of a fullerene $\mathrm{C}_{60}$ into a single-walled carbon nanotube. In an axially symmetric cylindrical polar coordinate system $(r, z)$, the centre of a fullerene $\mathrm{C}_{60}$ of radius $b$ is assumed to be located at $(0, Z)$ which can be inside or outside a carbon nanotube of semi-infinite length, centred around the positive $z$-axis and of radius $a$, as depicted in Figure 11. From Cox et al. [15] upon using equations (2) and (3), we find the potential of an atom on the carbon nanotube interacting with all atoms of the spherical fullerene to be given by

$$
P(\rho)=n_{f} \pi b A\left[\frac{(\rho+b)^{-4}-(\rho-b)^{-4}}{2 \rho}\right]-n_{f} \pi b B\left[\frac{(\rho+b)^{-10}-(\rho-b)^{-10}}{5 \rho}\right],
$$

where $\rho$ is the distance between the centre of the $\mathrm{C}_{60}$ molecule and an atom on the carbon nanotube, as shown in Figure 11. 
Figure 11 Geometry of a $\mathrm{C}_{60}$ fullerene entering a single-walled carbon nanotube

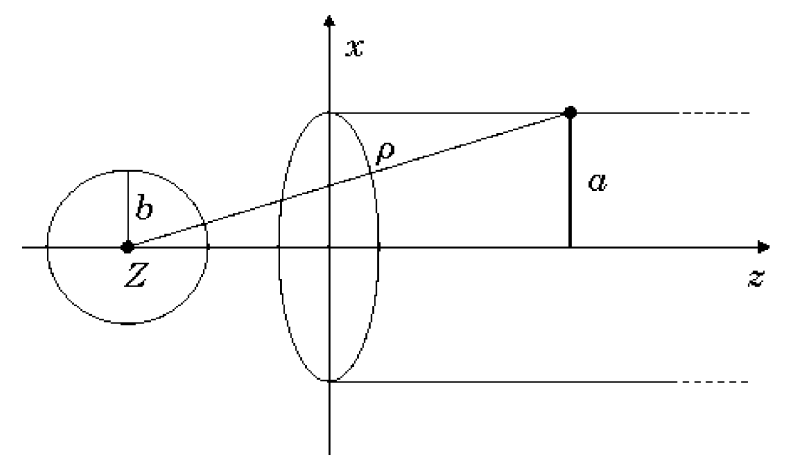

The van der Waals interaction force between the fullerene molecule and an atom on the tube is given by $F_{v d W}=-\nabla P$, thus from Figure 11 the axial force is of the form

$$
F_{z}=-\frac{(Z-z)}{\rho} \frac{\mathrm{d} P}{\mathrm{~d} \rho}
$$

As a result, the total axial force $F_{z}^{\text {tot }}(Z)$ of the entire carbon nanotube $(0 \leq z<\infty)$ interacting with the fullerene can be obtained by performing a surface integral of equation (16) over the tube, which upon simplifying gives

$$
F_{z}^{\text {tot }}(Z)=\frac{8 \pi^{2} n_{f} n_{g} a}{b^{4} \lambda^{3}}\left[A\left(1+\frac{2}{\lambda}\right)-\frac{B}{5 b^{6} \lambda^{3}}\left(5+\frac{80}{\lambda}+\frac{336}{\lambda^{2}}+\frac{512}{\lambda^{3}}+\frac{256}{\lambda^{4}}\right)\right],
$$

where $\lambda=\left(a^{2}-b^{2}+Z^{2}\right) / b^{2}$. In Figure 12, we plot equation (17) and find that there are at most two real roots $Z= \pm Z_{0}$ for equation $F_{z}{ }^{\text {tot }}(Z)=0$ and these roots only exist when the radius $a$ is less than some critical value $a_{0}$, where $b$ is given. In the case of a $\mathrm{C}_{60}$ fullerene $(b=3.55 \AA)$ we find $a_{0}=6.509 \AA$.

Figure 12 Force experienced by a $\mathrm{C}_{60}$ fullerene due to van der Waals interaction with a semi-infinite carbon nanotube

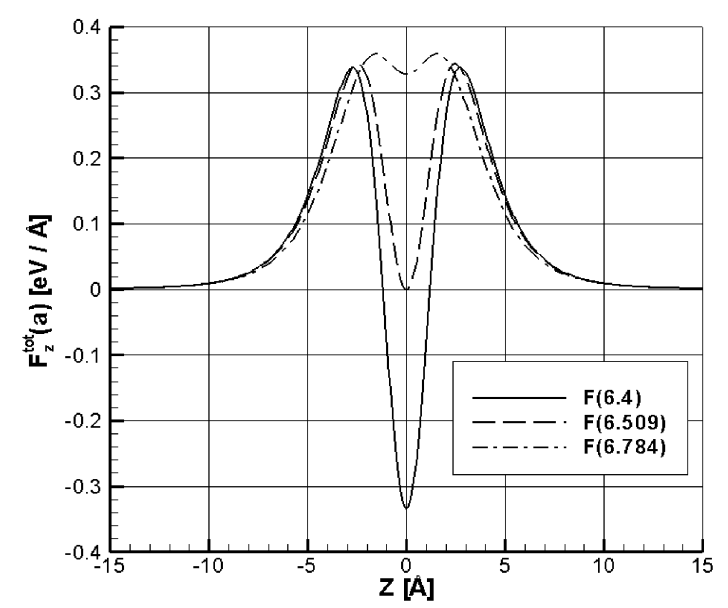


The integral of $F_{z}^{\text {tot }}(Z)$ represents the work imparted to the fullerene and equates directly to the kinetic energy. Therefore, the integral of $F_{z}^{\text {tot }}$ from $Z=-\infty$ to $Z_{0}$ represents the acceptance energy $\left(W_{a}\right)$ for the system and must be positive for a nanotube to accept a fullerene by a suction force alone. If $W_{a}$ is negative, then the magnitude of $W_{a}$ represents the initial kinetic energy needed by the fullerene in the form of the inbound initial velocity for it to be accepted into the nanotube. In Figure 13, we show the acceptance energy $W_{a}$ for a fullerene and a nanotube of radii in the range $6.1<a<6.5 \AA$. From the figure, $W_{a}=0$ when $a=6.338 \AA$ and nanotubes which are smaller than this will not accept $\mathrm{C}_{60}$ fullerenes by a suction force alone. This implies that a $(10,10)$ nanotube $(a=6.784 \AA)$ will accept a $C_{60}$ fullerene from rest, however a $(9,9)$ nanotube $(a=6.106 \AA)$ will not. The result of this model is in excellent agreement with Hodak and Girifalco [18] and Okada et al. [30].

Figure 13 Acceptance energy threshold for a $\mathrm{C}_{60}$ fullerene to be sucked into a carbon nanotube

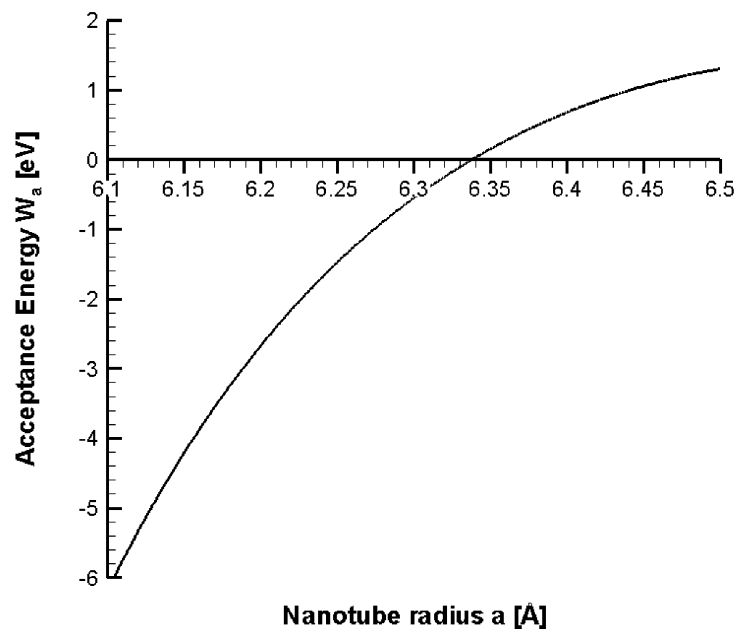

The suction energy $W$ for a fullerene, which is the total work performed by the van der Waals interactions on a $\mathrm{C}_{60}$ molecule entering a carbon nanotube, can be determined in a similar manner to $W_{a}$ but the integral is performed over the entire range $Z=-\infty$ to $\infty$. In Figure 14, we plot $W$ for a $\mathrm{C}_{60}$ molecule entering a nanotube with radii in the range $6<a<10 \AA$. We note that $W$ is positive when $a>6.27 \AA$ and has a maximum value of $W=3.242 \mathrm{eV}$ at $a=a_{\max }=6.783 \AA$. Accordingly, a $(10,10)$ carbon nanotube with $a=6.784 \AA$ is almost exactly the optimal size to maximise $W$ and therefore have a $\mathrm{C}_{60}$ fullerene accelerate to a maximum velocity upon entering the tube. Our model predicts that a $\mathrm{C}_{60}$ molecule in a $(10,10)$ carbon nanotube will accelerate to a velocity of $932 \mathrm{~m} / \mathrm{s}$; this result is in reasonable agreement with a molecular dynamics simulation of Qian et al. [14], which predicts a velocity of $840 \mathrm{~m} / \mathrm{s}$ for a $\mathrm{C}_{60}$ molecule entering the tube. 
Figure 14 Suction energy for a $\mathrm{C}_{60}$ fullerene entering a carbon nanotube

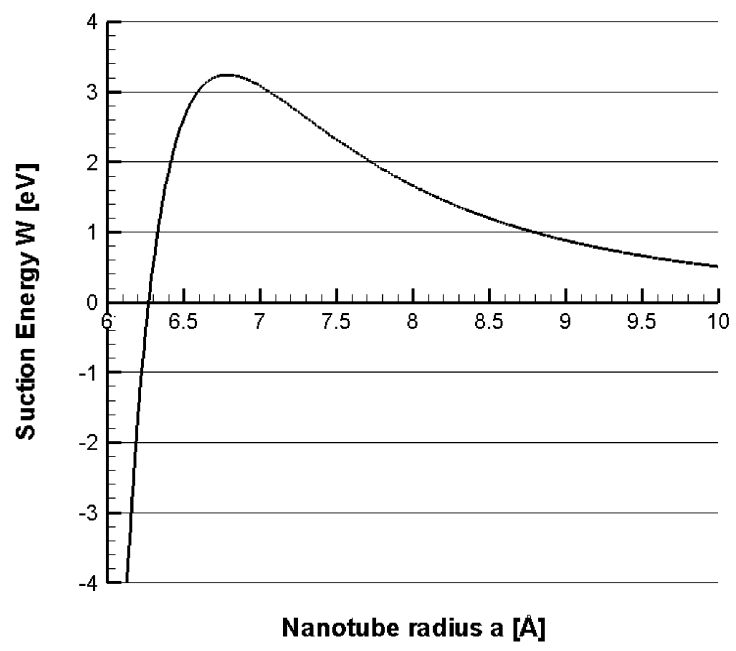

\subsection{Oscillatory behaviour}

In axially symmetric cylindrical polar coordinates $(r, z)$, we assume a $\mathrm{C}_{60}$ fullerene is located inside a carbon nanotube of length $2 L$, centred around the $z$-axis and of radius $a$. As shown in Figure 15, we assume that the centre of the $\mathrm{C}_{60}$ fullerene lies on the $z$-axis. This assumption is valid for a $(10,10)$ carbon nanotube, since from Section 2.2 the centre of the $\mathrm{C}_{60}$ fullerene will be forced on the tube axis to minimise the potential energy.

Figure 15 Geometry of the $\mathrm{C}_{60}$ fullerene oscillation

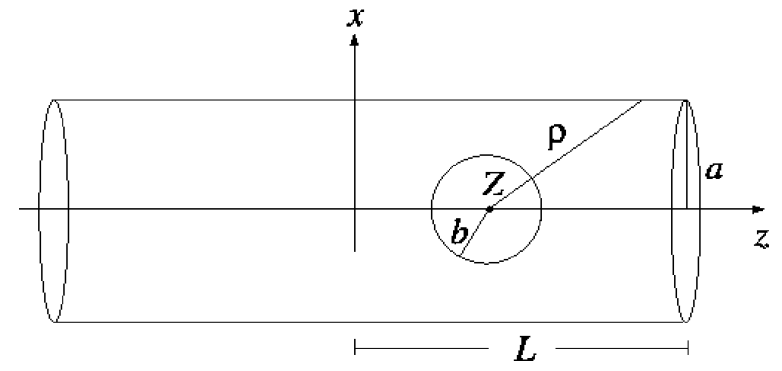

Again, we adopt Newton's second law to describe the oscillatory motion of the molecule inside a single-walled carbon nanotube, namely

$$
m \frac{\mathrm{d}^{2} Z}{\mathrm{~d} t^{2}}=F_{z}^{\mathrm{tot}}(Z),
$$

where $Z$ is the distance between the centres of the $\mathrm{C}_{60}$ fullerene and the carbon nanotube, $m$ is the total mass of the fullerene and due to the symmetry of the problem, we only consider the total axial van der Waals restoring force, $F_{z}^{\text {tot }}(Z)$, in the range $-L \leq Z \leq L$, which generates the oscillatory motion of the $\mathrm{C}_{60}$ fullerene. Following Cumings and Zettl [5], we neglect the frictional effect. Further, inside the carbon nanotube $(10,10)$, the 
$\mathrm{C}_{60}$ fullerene tends to move along the axial direction and not suffer rocking motion since its preferred location is on the $z$-axis. From Cox et al. $[15,16]$, the total axial force is shown to be of the form

$$
F_{z}^{\mathrm{tot}}(Z)=2 \pi a n_{g}\left[P\left(\rho_{2}\right)-P\left(\rho_{1}\right)\right]
$$

where $P(\rho)$ is defined by (15) and $\rho_{1}=\left[a^{2}+(Z+L)^{2}\right]^{1 / 2}$ and $\rho_{2}=\left[a^{2}+(Z-L)^{2}\right]^{1 / 2}$. In Figure 16, we plot $F_{z}^{\text {tot }}(Z)$ as given in equation (19) for a $(10,10)$ carbon nanotube of length $2 L=129 \AA$, and it can be seen that the force is close to zero everywhere except at the tube extremities. The pulse-like force at the tube ends operates to attract the $\mathrm{C}_{60}$ fullerene back towards the centre of the tube.

Figure 16 Total axial force for a $\mathrm{C}_{60}$ fullerene oscillating inside a $(10,10)$ carbon nanotube

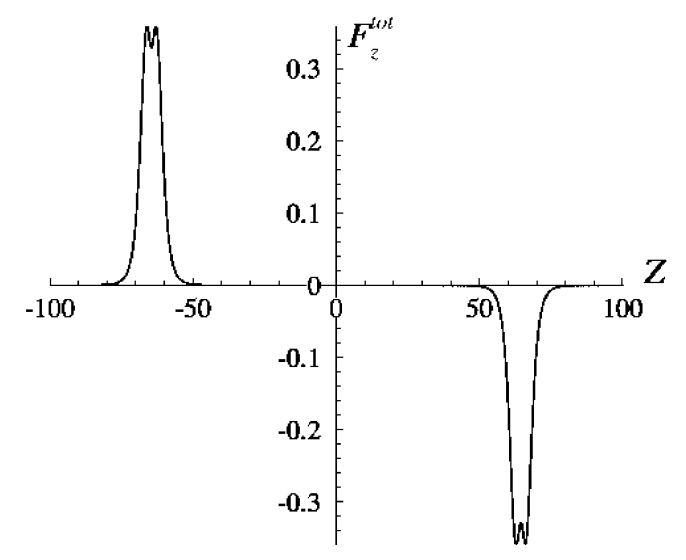

For $b<a<<2 L$, we find that $F_{z}^{\text {tot }}(Z)$ can be estimated by the Dirac delta function. As a result, equation (18) becomes

$$
m \frac{\mathrm{d}^{2} Z}{\mathrm{~d} t^{2}}=W[\delta(Z+L)-\delta(Z-L)]
$$

where $W$ is a constant representing the pulse strength or the work (energy) of the $\mathrm{C}_{60}$ fullerene, given by

$$
W=\int_{-\infty}^{0} F_{z}^{\text {tot }}(Z) \mathrm{d} Z=-\int_{0}^{\infty} F_{z}^{\text {tot }}(Z) \mathrm{d} Z
$$

By utilising the Heaviside step function, equation (18) can be integrated to give

$$
v=\frac{\mathrm{d} Z}{\mathrm{~d} t}=\left(\frac{2 W}{m}+v_{0}^{2}\right)^{1 / 2},
$$

for $-L \leq Z \leq L$, where $v_{0}$ is the initial velocity that the $\mathrm{C}_{60}$ fullerene is fired along the $z$-axis towards the open end of the tube in the positive $z$-direction. We note that the initial velocity $v_{0}$ is introduced for the case where the $\mathrm{C}_{60}$ fullerene is not sucked into the tube solely by the suction force due to the strong repulsion. From equation (22), it implies that the $\mathrm{C}_{60}$ fullerene travels inside the carbon nanotube at the constant speed $v$. As shown in Cox et al. [16], upon using equations (21) and (22) we obtain the velocity 
$v=932 \mathrm{~m} / \mathrm{s}$ for the case of the $\mathrm{C}_{60}$ fullerene initially at rest outside the carbon nanotube $(10,10)$ and the $\mathrm{C}_{60}$ fullerene gets sucked into the tube due to the attractive force alone. This obtained velocity agrees with the results of the molecular dynamics study by Qian et al. [14]. As such, we obtain the frequency $f=v /(4 L)=36.13 \mathrm{GHz}$. Figure 17 shows the variation of the oscillatory frequency with respect to the nanotube length. This result is in good agreement with the molecular dynamics simulation of Liu et al. [13], which confirms their findings that the shorter the carbon nanotube the higher the oscillatory frequency.

Figure 17 Variation of the oscillatory frequency of a $\mathrm{C}_{60}$ fullerene with respect to the length of a $(10,10)$ carbon nanotube

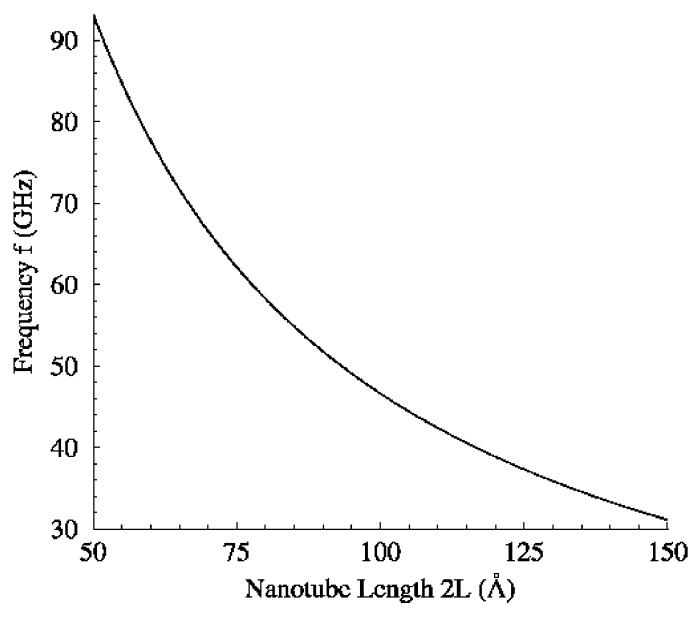

\section{$5 \mathrm{C}_{60^{-}}$- single-walled carbon nanotorus oscillators}

In order to study this problem, it is assumed that it is possible to close a $\mathrm{C}_{60}$-nanotube oscillator around onto itself so as to form the $\mathrm{C}_{60}$-nanotorus seamlessly. The vacuum effect, where a buckyball is sucked into one end of the nanotube [15,16], generating an initial velocity, is presumed to occur just prior to the closure of the nanotorus.

There are three forces which act on the orbiting buckyball inside a nanotorus; the van der Waals force (resulting from the Lennard-Jones potential), the centrifugal force; and the force of gravity, and each force has an associated potential energy function. Following the findings demonstrated by Cumings and Zettl [5], Hilder and Hill [19] assume that the friction is negligible when compared to the other remaining forces. Using the Lennard-Jones potential Hilder and Hill [19] derive the van der Waals interaction force, given as $F=-\nabla E(x, y, z)$, where $x, y, z$ refer to the coordinates of the buckyball and $E$ is the Lennard-Jones potential defined by equation (12). As the buckyball orbits inside the nanotorus it experiences a centrifugal force, which is the force experienced by a body spinning on an axis and is directed away from the centre of rotation. The centrifugal force is $F_{c}=-m R \omega^{2}$, where $m$ is the mass of the buckyball, with corresponding energy $E_{c}=-m R^{2} \omega^{2} / 2$. The buckyball also experiences a gravitational effect as it rotates, defined by $F_{g}=m g$, where $g$ is acceleration due to gravity $\left(9.81 \mathrm{~m} / \mathrm{s}^{2}\right)$. The corresponding potential energy can be written as $E_{g}=-m g h$, where $h$ is the height 
above some datum level and we assume the plane of the nanotorus is aligned to the horizontal. The total energy becomes $E_{t}=E+E_{c}+E_{g}$, and the position of the buckyball is located where this energy is a minimum.

Since gravity is found to be negligible, Newton's second law can be shown to reduce to

$$
\frac{\partial E}{\partial R}=m R \omega^{2}
$$

where $E$ is the Lennard-Jones energy given by equation (12), $m$ is the buckyball mass, $\omega$ is the angular velocity and $R$ is the distance from the centre of rotation to the centre of the rotating body $\left(R=c+\varepsilon \cos \varphi_{1}\right.$ and $\left.\varphi_{1}=0\right)$. By rearranging equation (23), a relationship between the angular velocity $\omega$ and the buckyball position $\varepsilon$ is determined. Figure 18 illustrates the angular velocity of a nanotorus created from closing a $(10,10)$ tube against the buckyball position, $\varepsilon$. At rest, the buckyball will locate itself to its equilibrium position $(\varepsilon=0.9 \AA$, shown in Figure 6$)$ and as a result there is no angular velocity until this position is reached. To move the buckyball from the equilibrium position, away from the centre of the nanotorus, an angular velocity must be applied. As shown in Figure 18, this velocity increases exponentially as the distance $\varepsilon$ increases. This shift away from the centre of rotation occurs for angular velocities in the gigahertz range, where the shift is greater as the angular velocity increases. For example, for the $(10,10)$ carbon nanotorus, a frequency of $34 \mathrm{GHz}$ moves the buckyball $0.4 \AA$ away from the equilibrium position, whereas a frequency of $150 \mathrm{GHz}$ moves the buckyball $1 \AA$ away. In contrast, in Section 4.2 (Cox et al. [16]) a frequency of $36.13 \mathrm{GHz}$ is found for the $\mathrm{C}_{60}$-nanotube oscillator comprised of a $(10,10)$ carbon nanotube.

Figure 18 Angular velocity of $(10,10)$ nanotorus against buckyball position $\varepsilon$

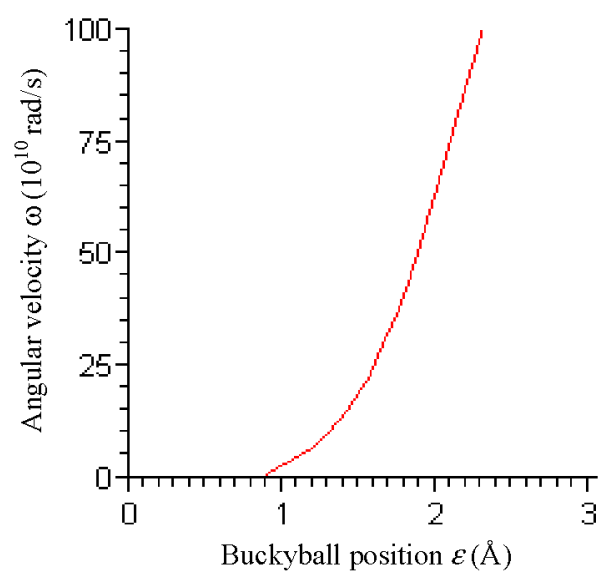

\section{Summary}

In this paper, we review the recent work of the authors on modelling the mechanisms of nano-oscillators, for which the oscillatory motion generates frequencies in the gigahertz range. Three different types of oscillators are presented; double-walled carbon nanotubes, $\mathrm{C}_{60}$-nanotube and $\mathrm{C}_{60}$-nanotorus oscillators. For all three configurations, we use the 
Lennard-Jones potential together with the continuum approach to determine the formal mathematical expressions for the energy and the van der Waals interaction force. We emphasise that the major contribution of the authors in this area is the use of elementary mechanics and classical applied mathematics to formulate explicit analytical expressions and ideal model behaviour in a scientific context previously only elucidated through experiments and molecular dynamics studies.

For double-walled carbon nanotube oscillators, we determine mathematical expressions that can be used to describe the force distribution and oscillation behaviour for double-walled carbon nanotubes of arbitrary lengths $2 L_{1}$ and $2 L_{2}$ for the inner and outer tubes, respectively. The oscillatory behaviour is most likely to occur when the inner tube end coincides with the outer tube end due to the strong repulsive force. We note that the period of these forces act when the inner tube position is in the regions $\beta_{1}-\beta_{2}$ and $\beta_{3}-\beta_{4}$, where $\beta_{1}=-L_{1}-L_{2}, \beta_{2}=L_{1}-L_{2}, \beta_{3}=L_{1}+L_{2}$, and $\beta_{4}=-L_{1}+L_{2}$. Furthermore, it can be seen that if we pull the inner tube out with the extrusion distance $d<2 L_{1}$ and release it, the inner tube will oscillate inside the outer tube without escape. This is because the strong attractive forces at the ends of the outer tube maintain the oscillatory behaviour. We observe that in general the longer the extrusion distance and the shorter the inner tube length, the higher the oscillatory frequency.

For the case of a $\mathrm{C}_{60}$ fullerene inside a single-walled carbon nanotube, we prescribe the potential energy of the system and the results show that as the tube radius gets larger, the $\mathrm{C}_{60}$ fullerene is likely to be closer to the tube wall. For a $(10,10)$ carbon nanotube, we find that the centre of the buckyball is located at the centre of the cross-section of the tube due to this being the position of minimum energy. These results are in excellent agreement with Girifalco et al. [17] and Hodak and Girifalco [18]. For the suction of $\mathrm{C}_{60}$ fullerenes into single-walled carbon nanotubes, we find that the carbon nanotube with radius $a>6.338 \AA$ will suck in the $\mathrm{C}_{60}$ molecule from rest. For smaller tubes, an initial velocity is required for a buckyball to penetrate into the tube. We further find that a $\mathrm{C}_{60}$ fullerene admits the maximum velocity when it enters a carbon naotube with radius $a=6.783 \AA$. In terms of the oscillatory behaviour of a $\mathrm{C}_{60}$ fullerene inside a single-walled carbon nanotube, we find that the interaction force is close to zero everywhere except at the tube extremities, where two pulse-like forces operate to keep the $\mathrm{C}_{60}$ fullerene oscillating between both ends. We use the Dirac delta function to capture this essential phenomena and find that our mathematical model predicts the velocity of the $\mathrm{C}_{60}$ fullerene and the gigahertz oscillation frequency well in agreement with molecular dynamics simulations of Liu et al. [13] and Qian et al. [14].

For the $\mathrm{C}_{60}$ fullerene orbiting inside a horizontally inclined carbon nanotorus, we find that the effect of gravity is considerably less than that arising from the Lennard-Jones interaction energy and centrifugal force. Similar to the $\mathrm{C}_{60}$-nanotube oscillator, we find that as the nanotorus tube radius increases the buckyball moves close to the tube wall. The addition of the centrifugal force causes the buckyball to move closer to the tube wall and further from the centre of rotation. As the angular velocity increases this effect is increased. Frequencies are in the gigahertz range and values as high as $150 \mathrm{GHz}$ can be reached by a change of buckyball position of $1 \AA$ without the need for any structural change. We note that the $\mathrm{C}_{60}$-nanotorus oscillator is speculative in nature and presents exciting possibilities, however, there are still numerous practical challenges that need to be overcome before the $\mathrm{C}_{60}$-nanotorus oscillator can be realised. 


\section{Acknowledgements}

The authors are grateful to the Australian Research Council for support through the Discovery Project Scheme and the provision of an Australian Postdoctoral Fellowship for NT and an Australian Professorial Fellowship for JMH.

\section{References}

1 Dresselhaus, M.S., Dresselhaus, G. and Eklund, P.C. (1996) Science of Fullerenes and Carbon Nanotubes, Academic Press, San Diego.

2 Harris, P.J.F. (1999) Carbon Nanotubes and Related Structures: New Materials for the Twenty-first Century, Cambridge University Press, England.

3 Rao, C.N.R., Satishkumar, B.C., Govindaraj, A. and Nath, M. (2001) 'Nanotubes', ChemPhysChem, Vol. 2, pp.78-105.

4 Qian, D., Wagner, G.J., Liu, W.K., Yu, M.F. and Ruoff, R.S. (2002) 'Mechanics of carbon nanotubes', Appl. Mech. Rev., Vol. 55, pp.495-533.

5 Cumings, J. and Zettl, A. (2000) 'Low-friction nanoscale linear bearing realized from multi-walled carbon nanotubes', Science, Vol. 289, pp.602-604.

$6 \mathrm{Yu}, \mathrm{M}-\mathrm{F}$., Yakobson, B.I. and Ruoff, R.S. (2000) 'Controlled sliding and pullout of nested shells in individual multiwalled carbon nanotubes', J. Phys. Chem. B, Vol. 104, pp.8764-8767.

7 Zheng, Q. and Jiang, Q. (2002) 'Multiwalled carbon nanotubes as gigahertz oscillators', Phys. Rev. Lett., Vol. 88, pp.045503.

8 Zheng, Q., Liu J.Z. and Jiang, Q. (2002) 'Excess van der Waals interaction energy of a multiwalled carbon nanotube with an extruded core and the induced core oscillation', Phys. Rev. B, Vol. 65, pp.245409.

9 Legoas, S.B., Coluci, V.R., Braga, S.F., Coura, P.Z., Dantas, S.O. and Galvão, D.S. (2003) 'Molecular dynamics simulations of carbon nanotubes as gigahertz oscillators', Phys. Rev. Lett., Vol. 90, pp.055504.

10 Rivera, J.L., McCabe, C. and Cumming, P.T. (2003) 'Oscillatory behavior of doublewalled nanotubes under extension: a simple nanoscale damped spring', Nano Lett., Vol. 3, pp.1001-1005.

11 Rivera, J.L., McCabe, C. and Cumming, P.T. (2005) 'The oscillatory damped behavior of incommensurate double-walled carbon nanotubes', Nanotechnology, Vol. 16, pp.186-198.

12 Baowan, D. and Hill, J.M. (2007) 'Accurate expressions for the force distribution for double-walled carbon nanotubes', Z. Angew. Math. Phys. (ZAMP), Vol. 58, pp.857-875, DOI: 10.1007/s00033-006-6098-z.

13 Liu, P., Zhang, Y.W. and Lu, C. (2005) 'Oscillatory behavior of C60-nanotube oscillators: a molecular-dynamics study', J. Appl. Phys., Vol. 97, pp.094313.

14 Qian, D., Liu, W.K. and Ruoff, R.S. (2001) 'Mechanics of $\mathrm{C}_{60}$ in nanotubes', J. Phys. Chem. B, Vol. 105, pp.10753-10758.

15 Cox, B.J., Thamwattana, N. and Hill, J.M. (2007) 'Mechanics of atoms and fullerenes in single-walled carbon nanotubes. I. Acceptance and suction energies', Proc. $R$. Soc. London, A, Vol. 463, pp.461-476.

16 Cox, B.J., Thamwattana, N. and Hill, J.M. (2007) 'Mechanics of atoms and fullerenes in single-walled carbon nanotubes. II. Oscillatory behaviour', Proc. R. Soc. London, A, Vol. 463, pp.477-494.

17 Girifalco, L.A., Hodak, M. and Lee, R.S. (2000) 'Carbon nanotubes, buckyballs, ropes, and a universal graphitic potential', Phys. Rev. B, Vol. 62, pp.13104-13110. 
18 Hodak, M. and Girifalco, L.A. (2001) 'Fullerenes inside carbon nanotubes and multi-walled carbon nanotubes: optimum and maximum sizes', Chem. Phys. Lett., Vol. 350, pp.405-411.

19 Hilder, T.A. and Hill, J.M. (2007) 'Orbiting atoms and $\mathrm{C}_{60}$ fullerenes inside carbon nanotori', J. Appl. Phys., Vol. 75, No. 12, pp.125415.

20 Liu, J., Dai, H., Hafner, J.H., Colbert, D.T. and Smalley, R.E. (1997) 'Fullerene crop circles', Nature, Vol. 385, pp.780, 781.

21 Martel, R., Shea, H.R. and Avouris, P. (1999) 'Rings of single-walled carbon nanotubes', Nature, Vol. 398, pp.299.

22 Wang, Y., Tománek, D. and Bertsch, G.F. (1991) 'Stiffness of a solid composed of $\mathrm{C}_{60}$ clusters', Phys. Rev. B, Vol. 44, pp.6562-6565.

23 Rieth, M. (2003) Nano-Engineering in Science and Technology: An Introduction to the World of Nano-Design, Series on the Foundations of Natural Science and Technology - Vol. 6, World Scientific, Singapore, pp.7-66.

24 Girifalco, L.A. (1992) 'Molecular properties of $\mathrm{C}_{60}$ in the gas and solid phases', J. Phys. Chem., Vol. 96, pp.858-861.

25 Henrard, L., Hernández, E., Bernier, P. and Rubio, A. (1999) 'van der Waals interaction in nanotube bundles: consequences on vibrational modes', Phys. Rev. B, Vol. 60, pp.R8521-R8524.

26 Gradshteyn, I.S. and Ryzhik, I.M. (2000) Table of Integrals Series and Products, 6th ed., Academic Press, San Diego.

27 Bailey, W.N. (1972) Generalized Hypergeometric Series, Hafner Publishing, New York.

28 Burchnall, J.L. and Chaundy, T.W. (1940) 'Expansions of Appell's double hypergeometric functions', Q. J. Math., Vol. OS-11, pp.249-270.

29 Ma, C.C., Zhao, Y., Yam, C.Y., Chen, G. and Jiang, Q. (2005) 'A tribological study of double-walled and triple-walled carbon nanotube oscillators', Nanotechnology, Vol. 16, pp.1253-1264.

30 Okada, S., Saito, S. and Oshiyama, A. (2001) 'Energetics and electronic structures of encapsulated $\mathrm{C}_{60}$ in a carbon nanotube', Phys. Rev. Lett., Vol. 86, pp.3835-3838. 